\title{
Memoria histórica y olvido en la enseñanza de la historia: los casos de Japón e Italia*
}

\section{Historical memory and forgetting in the teaching of history: the case of Japan and Italy}

\section{Emilio José Delgado-Algarra (1) Jesús Estepa-Giménez (2)}

(1) Universidad Loyola Andalucía (Sevilla)

(2) Universidad de Huelva

Resumen: En el presente artículo, se revisan algunas investigaciones y publicaciones relevantes donde se analizan aspectos vinculados con la inclusión de la memoria en la enseñanza de la historia (en los casos de Italia y, en mayor medida, Japón) y la conexión existente entre política y gestión de la memoria. Respecto a este último asunto, se evidencia la importancia de la política en relación a la selección y los olvidos en la memoria institucional y cómo los rituales conmemorativos o la construcción de una historia oficial implican un posicionamiento respecto a la memoria. Se observan posicionamientos políticos que determinan la selección de la memoria en la Historia oficial que, al mismo tiempo, es enseñada en los centros educativos.

Palabras clave: Enseñanza de la Historia, memoria histórica, gestión política de la memoria, Japón, Italia.

Abstract: In this paper, we review some researches and relevant publications where aspects related to the inclusion of memory in the teaching of history (in the cases of Italy and, further, Japan) and the connection between politics and memory management are analyzed. Regarding this last issue, it is evidenced the importance of policy regarding the selection and forgetting in the institutional memory and how the commemorative rituals or the construction of an official history involves positioning regarding the memory. Finally, political positions that determine the selection of the memory in the official history which, at the same time, is taught in schools, are observed.

Keywords: Teaching of History, historical memory, political management of memory, Japan, Italy.

(Fecha de recepción: noviembre, 2014, y de aceptación: septiembre, 2015)

DOI: 10.7203/DCES.29.4357

\footnotetext{
* El presente artículo tiene su origen en la Tesis Doctoral Educación para la ciudadanía en la enseñanza de las Ciencias Sociales y su vinculación con las dimensiones de la memoria: Estudio de caso en ESO dirigida por el Dr. Jesús Estepa Giménez (UHU) y el Dr. Francisco Florentino García Pérez (US), en la Universidad de Huelva.
} 


\section{Introducción: el olvido como fenómeno individual, colectivo e institucional}

No existe un posicionamiento universal en torno al papel del olvido en los fenómenos de cohesión social. El olvido, a corto plazo, puede convertirse en una elección racional cuando los grupos radicales hacen uso de los traumas para continuar con el conflicto. Esto se debe a que este olvido, a corto plazo, nos libra del pasado y nos permite construir unas nuevas bases (Lerner, 2007). Sin embargo, cuando se aparca la fuerza emocional implícita en los recuerdos y surgen cuestiones en torno a la selección mnemónica, el olvido deja de ser una opción satisfactoria en relación con la educación y una ciudadanía crítica y democrática. En otras palabras, cuando una institución democrática apuesta por el olvido como vía para "aparcar rencores" con el objetivo de fomentar la cohesión social, apuesta por una medida caduca donde se vulnera la libertad del individuo y su autodeterminación tanto en el plano individual como en el plano colectivo; sin embargo, cabe matizar que cuando una institución apuesta abiertamente por la selección de una memoria oficial, lo hace atendiendo sus propios intereses partidistas o ideológicos.

En el seno de esta disyuntiva, Todorov (2012) hace referencia a las trampas relacionadas con la selección y con los valores atribuidos a los recuerdos como posibilidad enmarcable en el ámbito de los "deberes de la memoria", lo que llega a convertir a los protagonistas de los acontecimientos en héroes o villanos en función de lo que se decide recordar y lo que se decide olvidar. Por otro lado, cuando se opta por el olvido, es decir, cuando eliminan eventos para minimizar las connotaciones subjetivas que puedan llevar a conductas pasionales, se entra en el ámbito de lo que Rinaldi (2008) denomina "deber del olvido", un deber que busca generar una distancia para que la memoria pueda ser tenida en cuenta como fuente de la construcción historiográfica.

El olvido adquiere un papel de gran importancia dado que aunque se opte por el recuerdo, toda selección de la memoria implica un olvido más o menos intencionado. Dicho olvido, por su parte, se mueve en tres planos fundamentales: el plano individual, el plano colectivo y el plano institucional u oficial. En un plano individual, De María (2006) hace referencia al ejemplo del prisionero de guerra que olvida episodios traumáticos o al del fascista que, tras el cambio de régimen, olvida sus momentos de mayor implicación con el mismo. Además, existe una íntima relación entre la memoria individual y la colectiva, en el plano colectivo, Oliva (2003) toma como ejemplo el olvido colectivo que se ha dado en zonas italianas donde se apoyó el fascismo, llegando al punto de alterar la memoria para que se recuerden esas zonas por su lucha antifascista. Finalmente, en el plano institucional, Mosse (1974) habla de cancelación de la memoria como un fenómeno protagonizado por los estados que buscan crear una determinada identidad nacional; 
para ello se elimina cualquier rastro mnemónico que pueda convertirse en un obstáculo para la nacionalización de las masas.

\section{Estado de la cuestión: la patrimonialización de la memoria y los retos de la escuela democrática}

Uno de los grandes limitantes de las potencialidades de la inclusión de la memoria histórica y social en la enseñanza de la historia se encuentra en que, desde la historia, en ocasiones, determinados aspectos de la memoria (vinculados con la construcción de una identidad concreta) adquieren un carácter cuasi mitológico (Benjamin, 2007). Como indica Kingman (2012), existe una fuerte tendencia hacia la patrimonialización de la memoria social, permitiendo con ello un rescate del inventario mnemónico en peligro de desaparición; pero corriendo el riesgo de que pueda ser banalizada e incorporada en forma de archivo muerto o de celebración. En este sentido, este mismo autor, indica la necesidad de distinguir entre una memoria patrimonializada o archivada, inconexa con la realidad social, y una memoria crítica, conectada con pasado, presente y futuro; siendo este último modelo de memoria clave para el establecimiento de un diálogo con la Historia y su enseñanza. En palabras de Aranzadi (2009), estas conmemoraciones promovidas por el gobierno de los Estados "no son nunca recuerdo o memoria fiel del pasado, sino un estímu- lo ideológicamente dirigido de la imaginación de los individuos, incitación a superponer sobre la memoria individual una interpretación políticamente orientada del recuerdo" (2009: 162)

Tanto los contenidos y la metodología usada para la enseñanza de la Historia como la aprobación y selección de libros de texto del área responden a criterios ideológicos. Ya sea por exceso, defecto o alteración, la memoria histórica presente en la enseñanza de la Historia puede reflejar intereses vinculados con el propio proceso identitario de un estado-nación. En este sentido, desde los sistemas democráticos, los partidos políticos que gobiernan, llevan a cabo mecanismos de control social a través de una interpretación del pasado que puede extrapolarse a la enseñanza de la historia en los centros educativos (Delgado-Algarra, 2014). Frente a este asunto, Raimundo Cuesta (2006), en defensa de una didáctica crítica, afirma que el uso público de la memoria histórica en la escuela implica la promoción de una "contramemoria crítica" que debe realizarse mediante la promoción de un conjunto de prácticas pedagógicas que generen conciencia social frente a problemas actuales e históricos. Pese a ello, la institución educativa se enfrenta al problema de la adopción de una doble postura frente a la memoria. En otras palabras, como indica Rinaldi (2008), la escuela debe asumir un reto de extrema importancia en cuanto a la enseñanza de la Historia: debe esforzarse por transmitir y procesar la memoria; pero a su vez debe esforzarse por atajar los efec- 
tos negativos y dudosos derivados de los excesos de la memoria.

Durante la I Guerra Mundial los dirigentes políticos de los Estados se dieron cuenta de que los libros de texto podían servir como herramienta de adoctrinamiento, lo cual se radicalizó en la II Guerra Mundial hasta el punto de la promoción de libros de texto para la demonización de los países enemigos; estos ejemplos demuestran que el contenido de los libros de texto de Historia ha estado tradicionalmente muy vinculado al poder político. Tanto el adoctrinamiento unilateral en relación con la memoria como la eliminación de la misma genera un rechazo a la pluralidad de memorias, de manera que la existencia de recuerdos opuestos sobre la guerra se convierte en un obstáculo para la reconciliación. Como indica Rinaldi (2008), la escuela debe transmitir y procesar la memoria histórica evitando los sesgos ideológicos o de adoctrinamiento; ya que, debido al carácter subjetivo y parcial de la memoria, surgen "memorias divididas" o "memorias en conflicto" que, como afirma Lerner (2007), pueden llevarnos a vivir el presente como continuación natural de una guerra, potenciando el surgimiento de nuevos enemigos a raíz de los enemigos históricos (obsesión de la memoria); sin embargo, el olvido, como señala este mismo autor, deja evidencias de sus limitaciones una vez se supera la retórica de los valores que impregnan la memoria y nos preguntamos por el problema de su selección.

Junto a la "aceptación" de la existencia de una serie de memorias en conflicto y la "negación" de la memoria, existe una tercera posibilidad, la de "conciliación" de dichas memorias, un proceso arduo y difícil dado que la memoria está cargada de valores muy diversos y difíciles de conciliar. Como ejemplo significativo de conciliación de las memorias cabe destacar el de la "Truth and Reconciliation Commision" (Brahm, 2007) en Sudáfrica donde, en lugar de apostar por el olvido o por la simple aceptación de la existencia de conflictos, se produce un acercamiento de la memoria de verdugos y víctimas del pasado racista del país a través de la comparación con el objeto de consolidar la verdad histórica, superar la fijación en esa etapa y transformar la memoria en conflicto en memoria conciliada. Existen otros ejemplos, como el de la "Comisión Nacional de Verdad y Reconciliación" de Chile en 1991; sin embargo, en este caso, cabe matizar que las carencias de representación de la izquierda en la misma llevó a que la violencia de la derecha se presentase brevemente y como respuesta a una crisis generala por la izquierda (Oteíza, 2011).

Volviendo al caso de Europa, tras la salida de los reyes de la mayor parte del continente, y siguiendo la tendencia iniciada durante la I Guerra Mundial, líderes mesiánicos como Hitler, Lenin o Stalin pasaron a gobernar y a ejercer una fuerte influencia en los contenidos de los libros de texto con objeto de construir concepciones dogmáticas de ciudadanía nacional, usándose como arma contra el enemigo. Esta situación trató de solventarse sin éxito por la Liga de las Naciones, hasta que, tras las II Guerra 
Mundial, fue la UNESCO quien tomó las riendas del problema de la xenofobia en los libros de texto con el apoyo de profesores. En relación con esto, Lässig (2009) nos habla de la influencia que tuvo el historiador alemán Georg Eckert, quien inmediatamente después de la guerra, organizó una serie de conferencias para buscar la reconciliación con los antiguos enemigos de Alemania (Francia, Gran Bretaña, Polonia, Israel) a través de textos compartidos. Frente a esta búsqueda de la cohesión social, cabe matizar que "para los editores, elaborar y publicar un libro de texto es un desafí, pues deben hacer frente a las exigencias de los currículos escolares y a sus posibles interpretaciones, a la tradición que quieren mantener unos docentes y al cambio que exigen otros, a las disyuntivas que plantean las tensiones entre un producto rentable que se venda bien y el servicio educativo que pide calidad pedagógica y de contenido, a los intereses de distintos grupos sociales y al cuidado en el tratamiento de los géneros, de los grupos étnicos, de los movimientos politicos, religiosos, ambientales, y de otros tipos" (Mejía, 2009: 488). Como podremos entender, el interés que suscitan los libros de texto como recursos para la socialización de futuros ciudadanos adultos, se hace especialmente palpable en el caso de los libros de texto de Ciencias Sociales, Geografía e Historia; cuyo contenido curricular y su uso en el aula pueden configurar una realidad social determinada por la fuerza política dominante.

\section{Problemática}

Problema central

- ¿Qué convergencias existen en relación con la gestión política de la memoria y su inclusión en la enseñanza de la Historia en Japón e Italia?

Problemas específicos

- ¿Cuál es papel de la política en cuanto a la gestión de la memoria?

- ¿Cómo se incluye la memoria y el olvido en la enseñanza de la Historia en Japón e Italia?

\section{Objetivos}

Objetivo general

- Analizar los paralelismos y analogías en relación con la gestión política de la memoria y su inclusión en la enseñanza de la Historia de Japón e Italia.

Objetivos específicos

- Comprender el papel de la política en la gestión de la memoria.

-Comprender los condicionantes de la inclusión de la memoria y el olvido en la enseñanza de la Historia en Japón e Italia.

\section{Métodos e instrumentos}

La presente investigación pretende establecer un análisis comparativo entre los resultados de algunas investigaciones e iniciativas de reconciliación de memorias fragmentadas en la enseñanza de la Historia en Japón e Italia, respectivamente. A través de una metodología cualitativa, se llevará a cabo una 
revisión de diferentes investigaciones cuyas conclusiones serán contrastadas partiendo de algunos aspectos iniciados en la Tesis Doctoral de Delgado-Algarra (2014). Todo ello se realizará atendiendo a dos bloques fundamentales:

a) El papel que tiene la política en relación con la gestión de la memoria.

b) Cómo se introduce la memoria (y el olvido) en la enseñanza de la Historia en Italia y Japón.

\section{Muestra}

La muestra es de tipo bibliográfica. Para ello, en el caso de Japón, hemos extraído algunos de los aspectos clave de los resultados del Proyecto de Investigación "Divided Memories: History Textbooks and East Asian History", coordinado por los investigadores Gi-Wook Shin y Daniel Sneider (2011); acuerdos y desacuerdos de las iniciativas intergubernamentales para la reconciliación de Japón, Corea y China del Comité de Investigación de Historia Conjunta Japón - República de Corea (2001) y del Comité de Investigación de Historia Conjunta Japón - China (2006); y las contranarrativas revisionistas de la "Sociedad Japonesa para la Reforma de los Libros de Texto de Historia" (新し い歴史教科書をつくる会); además de las aportaciones de otros investigadores. En el caso de Italia, hemos seleccionado algunas conclusiones de parte de la actividad investigadora producida en torno “Clio'92: Associazione di inseg- nanti e ricercatori sulla didacttica della storia" presidida por Ivo Mattozzi.

\section{Resultados}

El caso de Japón

Memorias fragmentadas: consensos $y$ controversias

En el seno del Proyecto de Investigación "Divided Memories: History Textbooks and East Asian History" se analiza cómo se incluye la memoria en los libros de texto de Historia de uso mayoritario de Japón, Corea, China y Estados Unidos. Para ello, se gira en torno a una serie de bloques, de los cuales, por motivos de espacio, hemos seleccionado tres. En cuanto a la masacre de Nanjing, en los libros de Historia, se puede observar una sucesión de datos y fechas que se centra en las grandes batallas y coaliciones, como la coalición anti-japonesa de los partidos Nacionalista y Comunista de China, en estrategias militares de ocupación desde un punto de vista muy general, hablando en términos de "guerra entre tropas". Únicamente un libro hace referencia directa a los asesinatos y violaciones que se dieron en este periodo. En relación con las labores forzadas (incluyendo las "confort women"), los textos japoneses hacen referencia a dichas labores sin profundizar en ellas y a los movimientos de resistencia a los que tuvo que enfrentarse Japón. En todos se hace referencia a la imposición del idioma japonés y de la visita a los santuarios; sin embargo, únicamente en uno se alude directamente a las 
"confort women". Finalmente, en cuanto a los Juicios de los Crímenes de Guerra de Tokio, en todos los libros, se incluyen referencias a la rendición de Japón, al arresto de militares y oficiales, y a cómo 28 de los cuales fueron declarados "criminales de clase A"; haciéndose referencia a las ejecuciones producidas a raíz de los juicios, pero no indagando en el por qué. En uno de los libros, se indica explícitamente que los resultados de los Juicios estuvieron determinados por la política de ocupación de los EEUU y se añade que, en los mencionados juicios, se excluyeron los "bombardeos indiscriminados" a Japón, poniendo de ejemplo las bombas de Hiroshima y Nagasaki.

Con el Comité de Investigación de Historia Conjunta Japón - República de Corea (2001) y el Comité de Investigación de Historia Conjunta de Japón - China (2006), ambos de tipo gubernamental y formados por historiadores de los países participantes, se buscó un acercamiento de las respectivas memorias fragmentadas. Resultado de la labor de estos comités, se aceptaron algunos hechos básicos pese a que no se llegó a un acuerdo respecto a puntos más concretos vinculados con las víctimas y las intencionalidades subyacentes en las acciones militares. En este sentido, Haruo Tohmatsu (2011), ha indicado las cuestiones históricas que a día de hoy son aceptadas y los aspectos que son divergentes entre sí (tabla 1), lo cual se refleja en los libros de texto de Historia de Japón (Jp.), Corea (Co.) y China (Ch.).

\section{Tabla I. Hechos aceptados y puntos de controversia en los libros de Japón, China y Corea}

\begin{tabular}{|c|c|c|}
\hline & Hechos básicos aceptados & Puntos de controversia \\
\hline $\begin{array}{l}\text { Masacre de Nanjing } \\
\text { (diciembre de 1937- } \\
\text { enero de 1938) }\end{array}$ & $\begin{array}{l}\text {-Comenzó el } 15 \text { de diciembre de } 1937 . \\
\text {-Militares japoneses asesinaron } \\
\text { a muchos prisioneros de guerra y } \\
\text { civiles. }\end{array}$ & $\begin{array}{l}\text {-Víctimas: } 200.000 \text { (Jp.) / } 300.000 \\
\text { (Ch.) } \\
\text {-Naturaleza de la agresión: no } \\
\text { premeditada (Jp.) / premeditada (Ch.) }\end{array}$ \\
\hline $\begin{array}{l}\text { Labores forzadas } \\
\text { (incluyendo las } \\
\text { "confort women") }\end{array}$ & $\begin{array}{l}\text {-Condiciones inhumanas de } \\
\text { trabajadores chinos, coreanos, } \\
\text { taiwaneses, aliados y otros. } \\
\text {-Existencia de las "confort women" }\end{array}$ & $\begin{array}{l}\text {-Carácter voluntario de las "confort } \\
\text { women": sirvieron a los militares } \\
\text { voluntariamente (Jp.) / sirvieron de } \\
\text { manera obligada (Co.) }\end{array}$ \\
\hline $\begin{array}{l}\text { Juicios de los } \\
\text { Crímenes de } \\
\text { Guerra de Tokyo } \\
(1946-1948)\end{array}$ & $\begin{array}{l}\text { - } 28 \text { militares y oficiales fueron } \\
\text { declarados criminales de guerra clase } \\
\text { A; } 7 \text { de ellos fueron condenados a } \\
\text { muerte y el resto fue a prisión }\end{array}$ & $\begin{array}{l}\text {-Estados Unidos eximieron a otros } \\
\text { responsables y al Emperador Hirohito } \\
\text { (Ch.) } \\
\text {-Los Juicios de Tokio formaron parte } \\
\text { del plan de ocupación estadounidense; } \\
\text { pero no se hace crítica directa a su } \\
\text { legitimidad (Jp.) }\end{array}$ \\
\hline
\end{tabular}

Fuente: Elaboración propia a partir de Tohmatsu (2011) 
Estos resultados convergen con los estudios y análisis de otros autores. Nozaki (2001) ya destacó que en los libros de texto de Historia japoneses no suelen aparecer referencias a las "confort women"; Ienaga (1993) criticó la falta de remordimiento por el pasado que se reflejaba en los textos, haciendo alusión a las responsabilidades del Emperador Hirohito y la de los propios japoneses; por otro lado, Higurashi (2008) destacó que la ausencia de crítica a la legitimidad de los Juicios de Tokio se debe a que fueron asumidos como un castigo por parte de Japón debido a las atrocidades de la guerra, asegurándose con ello la reconciliación con los aliados. Este último aspecto es uno de los focos de interés de la revisionista "Sociedad Japonesa para la Reforma de los Libros de Texto de Historia" que, en palabras de uno de sus miembros, Fuijoka (2000), se vincula con la "Jigyaku rekishi-kan" o "visión masoquista de la Historia", una historia que, según él, fue impuesta por Estados Unidos. En relación con esto, el secretario general del sindicato docente "Internacional de Educación", Van Leeuwen (2007), indicó que "la dialéctica de patriotismo y humillación nacional carece de fundamento moral y hace un flaco favor a la juventud. La exactitud histórica es un requisito imprescindible para aprender del pasado y asentar el futuro en clave de ciudadanía y democracia" (2007: 5).

La Sociedad Japonesa para la Reforma de los Libros de Texto de Historia y su labor revisionista
Tokuji Kasahara (2007) señala que, a día de hoy, en los libros de texto predomina el intervencionismo estatal cuya tendencia implica el "suavizado" de los errores cometidos por parte de Japón; una situación que se asocia con la continuidad de la clase política desde la guerra y la reinterpretación de la historia derivada de las acciones de Japón en Asia durante la primera mitad del siglo XX, la cual queda plasmada en los textos de la "Sociedad Japonesa para la Reforma de los Libros de Texto de Historia" ("Atarashii Rekishi Kyōkasho o Tsukuru Kai", conocida habitualmente como "Tsukuru Kai").

Haciendo un breve repaso a algunos hechos significativos en cuanto a la gestión de la memoria en Japón, cabe mencionar las controversias generadas en relación con las declaraciones de oficialidad de las visitas del ex Primer Ministro Nakasone al Santuario de Yasukuni desde 1985 (Mochizuki, 2010) o el reconocimiento oficial del Kimigayo como himno nacional desde 1999 , un himno que se encontraba en desuso desde la II Guerra Mundial y que se incluiría en las ceremonias de graduación escolar desde 2006 (Delgado-Algarra, 2013). Esta última reforma fue foco de duras críticas y movilizaciones por un sector importante del profesorado y de sindicatos como la "Japan Teachers Union", cuyo representante en aquel momento, Yasuo Morikoshi, destacaba el carácter poco democrático de esta decisión que, según encuestas, contaba únicamente con el apoyo de un 4\%, de la ciudadanía. En el proceso de transición 
de lo político a lo escolar, en el año 2001, el Ministerio de Educación japonés (actualmente Ministerio de Educación, Cultura, Deporte, Ciencia y Tecnología) aprobó un "libro de texto revisado" por la Sociedad antes mencionada, que generó duras críticas de una parte de la ciudadanía de Japón, Corea del Sur y China debido a su carácter nacionalista, conservador y parcial (Beal, Nozaki y Yang, 2001; Nozaki, 2008; Mochizuki, 2010). Paradójicamente, todos sucede en el marco de Ley Fundamental de Educación (1947), que prohíbe al Gobierno "toda intervención excesiva" en la enseñanza.

Pese la autorización de los libros de texto de la Tsukuru Kai bajo el mandato del Partido Democrático Liberal, cabe matizar que su uso en las escuelas japonesas es extremadamente minoritario (menos del $0.5 \%$ ) y que, como demuestra la investigación realizada por Shin y Schneider (2011) el carácter aséptico de los contenidos de los libros de texto de Historia de uso mayoritario en Japón contrasta con el exceso de memoria que se produce en los textos escolares de la misma área en China (respecto a Japón) o con el patriotismo de los libros de texto de China y Estados Unidos. Sin embargo, pese a que dentro de las escuelas japonesas existe una tendencia a enseñar la historia de manera factual y acrítica (evitando polémicas o dañar sensibilidades), la prensa no japonesa suele cometer el error de criticar el carácter patriótico de los libros de texto de Historia japo- neses como si los libros de la Tsukuru Kai fuesen de uso extendido.

Sin embargo, pese al carácter aséptico de la enseñanza de la Historia en Japón, se ha ido observando un ligero incremento del nacionalismo en una parte de la juventud japonesa; un nacionalismo que, como indica Kayama (2002), carece de una verdadera conciencia histórica y parece querer evitar las emociones que suscitan las memorias en conflicto. Esta situación podría deberse a carencias que se dan en el análisis de la memoria dentro del curriculum escolar de Historia. Dicho de otra forma, y por poner un ejemplo, autores como Hisashi Yano (2007), comparan los trabajos forzados de coreanos y chinos en Japón con el caso alemán. Planteamientos como éste pueden generar un conflicto de memorias que, sin un análisis crítico a través de la enseñanza de una Historia con memoria, desembocan en una serie de lagunas en el desarrollo de la conciencia histórica. Para solventar los conflictos mnemónicos no analizados desde la escuela y las consecuentes deficiencias en la conciencia histórica, en algunos sectores de la juventud, se podrían estar incrementando actitudes nacionalistas y sesgadas, una situación que lastra cualquier deseo de construcción de una ciudadanía comprometida, crítica y democrática.

El caso de Italia: la reinterpretación de la memoria y el olvido

A la hora de educar para la ciudadanía, en Italia, la creación de la asigna- 
tura dedicada específicamente a ello, no ha impedido que se mantenga una rigidez curricular de contenidos y una evaluación focalizada casi exclusivamente en la superación de un examen. Esta situación, como indica Brusa (2010), precisa de un cambio de los contenidos curriculares que se dan en la escuela de cara a buscar solución a los problemas del mundo actual. Por otro lado, debemos añadir el carácter maleable de la memoria colectiva por parte de políti$\cos$ y periodistas que buscan ajustar el pasado a determinados intereses ignorando las aportaciones científicas. En relación a esto, Pivato (2007) destaca que Italia es, probablemente, el único país del mundo en el que los ciudadanos jamás han estudiado la historia de la posguerra. Según Oliva (2003), la Resistencia es un recurso que los italianos usan para alejarse de la memoria vinculada con el apoyo al fascismo y para atenuar la sensación de haber perdido la guerra. Es por ello que, como indica, dicho autor, Italia no ha llegado a un acuerdo con su pasado, y esto se debe a casos como el de las ciudades en las cuales los italianos lucharon junto a Hitler (1940 - 1943), donde la memoria colectiva tiende enfocar su mirada en el papel de la ciudadanía en la guerra antifascista contra Hitler (1940 - 1945). Esta situación lleva a que las lápidas en memoria de los soldados que murieron en las campañas de los Balcanes o dispersos por Rusia se encuentren junto a las lápidas en memoria a los guerrilleros caídos y de las víctimas de los campos de concentración.
En otras palabras, a la hora de gestionar la memoria, se tienden a omitir sucesos o a reinterpretarlos en base a determinados intereses. Por ello, Ken Ishida (2007), cuando hace alusión a los crímenes de guerra de Japón e Italia (en particular los cometidos desde el comienzo del siglo XX en Corea, China, Libia y Etiopía), destaca las dificultades que tienen ambos países para desarrollar una memoria crítica frente a los crímenes cometidos y frente a las limitaciones del castigo a los criminales de guerra tras la Segunda Guerra Mundial; siendo estas limitaciones mucho mayores en el caso de Italia, lo cual, igualmente, se refleja en el tratamiento de la memoria en la enseñanza de la historia. Como afirma Vanzetto (s/f), la memoria pública es demasiado delicada como para ser confiada exclusivamente a la "ingeniería del consenso"; igualmente, añade, es necesaria una profunda revisión de la enseñanza de la historia en Italia, ya que el traspaso de masa de información enciclopedista de arriba abajo impide la representación de un pasado funcional de cara a la formación de un "buen ciudadano".

Ivo Mattozzi (2008), profesor de la Universidad de Bolonia, ha estado trabajando durante más de 30 años con profesores de historia e historiadores, y con fuentes de la memoria y testimonios orales. A raíz de ese trabajo, el autor se plantea cuatro problemas a razonar sobre la relación entre memoria y formación histórica: cómo incluir la memoria para beneficiar a la formación histórica, cómo la memoria puede ser un 
recurso o un estorbo para la formación histórica, cómo hacer que la formación histórica beneficie a las representaciones sobre rastros mnemónicos y cómo hacer que la formación histórica pueda volvernos competentes para criticar las representaciones sobre rastros mnemónicos. Todo eso teniendo en cuenta que, como indica Diez Gutiérrez (2013): "los texto escolares dominan el currículum (...) ayudan a construir el imaginario de las futuras generaciones" (2013: 24).

\section{Conclusiones}

Respecto al papel de la política en la gestión de la memoria, a lo largo del artículo hemos evidenciado su importancia en relación a la selección y los olvidos en la memoria institucional y cómo los rituales conmemorativos o la construcción de la historia oficial implican un posicionamiento respecto a la memoria (Estepa-Giménez y Delgado-Algarra, 2015). Tanto en el caso japonés, con el posicionamiento oficial respecto a la Masacre de Nanjing, las labores forzadas (incluidas las "confort women") y los Juicios de Tokio, como en el caso italiano, con la Resistencia y los crímenes de guerra, hemos observado posicionamientos políticos que determinan la selección de una memoria que se refleja en la Historia oficial que, a su vez, es enseñada en los centros educativos. Los textos escolares son un reflejo del posicionamiento de los partidos gobernantes; de este modo, mientras que en la enseñanza de la Historia de Italia se tiende a la omisión de episo- dios de la posguerra, en la enseñanza de la Historia de Japón se tiende a la "objetividad aséptica". En el caso de Japón, respecto al cual hemos decidido una mayor profundización, destacamos algunas iniciativas internacionales de acercamiento en la construcción de una historia común sin fisuras mnemónicas y de una aproximación hacia la confección de textos comunes con sus vecinos del este de Asia; sin embargo, siguen existiendo controversias en cuestiones concretas relacionadas con la acción imperialista de Japón sobre Asia durante el primer tercio del siglo XX.

Del análisis de estos dos casos podemos extraer también otras conclusiones didácticas más generales, como que la memoria histórica puede ser un excelente recurso en el aprendizaje de la Historia, sobre todo a partir de fuentes orales, ya que, como afirma Pagés (2008), puede permitir al alumnado obtener información directa sobre muchos aspectos de la historia reciente, tanto los que se refieren a memoria de lugares como a memoria de hechos traumáticos pasando por la memoria de hechos de la vida cotidiana vinculados con el trabajo, la vivienda, las migraciones, el estudio o el ocio (p.50). No obstante, para llevar a cabo un proceso didáctico crítico respecto a la memoria, se debe apostar por una escuela democrática donde el docente asuma un modelo de imparcialidad comprometida basado en la libertad dialéctica profesor - alumno (Kelly, 1986; Cuesta, 2011); sin embargo, otro de los retos a los que se enfrenta la escuela es el de 
paliar las incoherencias que, según diversas investigaciones, se producen entre los deseos del docente y su práctica (Tutiaux-Guillon, 2003). A todo ello, se suman tres factores limitantes a los que se debe atender a la hora de incluir la memoria en las clases de Historia (Hess, 2002, 2008): el miedo que en ocasiones tiene el profesorado novel a analizar críticamente temáticas controvertidas con sus alumnos, las concepciones docentes y el miedo a la reacción de la comunidad educativa. En esencia, como indican Pagés y Oller (2005), se deben incluir problemas relevantes, se deben usar métodos interactivos y se debe conectar pasado, presente y futuro de cara a promover la justicia social y eliminar los prejuicios en el alumnado; todo ello evitando la implementación de debates moralistas donde las respuestas predecibles aseguren un consenso acrítico del alumnado. Sin embargo, la escuela tiende a simplificar las problemáticas mediante la aplicación de "éticas blandas" que desemboquen en un "consenso feliz" (Bueno, 2007).

En definitiva, la escuela debe ser entendida como esfera pública que posibilite la formación de una opinión civil independiente del poder estatal a través del uso de estrategias deliberativas (Habermas, 1994; Martínez Bonafé, 2004; Cuesta, 2011), permitiendo con ello la rememoración del pasado desde el presente para poder actuar en el futuro. Así, pese a que observamos que se tiende a la "desmemoria" en la enseñanza de la historia, la Historia oficial que se refleja en el currículum de Historia está condi- cionada por las memoria oficial; evitándose abiertamente la inclusión de una didáctica crítica en relación con asuntos mnemónicos más allá de la búsqueda del consenso a través de un debate superficial donde se ponen en marcha escasas competencias críticas y autónomas respecto a las concepciones hegemónicas de la Historia; sin embargo, "no se trata de perder el tiempo con un argumentario de tópicos corporativos, sino de reflexionar sobre las armaduras éticas y politicas con las que una ciudadanía crítica debe dotarse" (López , 2008, p.41). Por otro lado, como indica Aranzadi (2009), la Historia siempre tiene algo de ideológico, especialmente cuando es considerada como una ciencia "objetiva del pasado", por lo que concebir una historia desmemoriada y objetiva como verdad absoluta es asumir como inalterable la imposición de una historia hegemónica, en consecuencia, la sustitución de la memoria individual por una memoria oficial.

\section{Referencias bibliográficas}

ARANZADI, J. (2009). Historia y nacionalismos en España hoy. En I. OLMOS y N. KEILHOLZ-RÜHLE (eds.), La cultura de la memoria histórica en España y Alemania (159172). Madrid: Iberoamericana.

BENJAMIN, W. (2007). El libro de los Pasajes. Madrid: Akal.

BRUSA, A (2010). Italia: la educación cívica, entre la utilización política y el trabajo en las escuelas. Íber, $\mathrm{n}^{\circ} 64$, 38-47. 
BUENO, G. (2007). La fe del ateo. Madrid: Editorial Temas de Hoy.

CUESTA, R. (2007). La enseñanza de la historia como contramemoria crítica. En S. LEONÉ y F. MENDIOLA (coords.), Voces e imágenes en la historia: fuentes orales y visuales: investigación histórica y renovación pedagógica. Actas del Congreso Internacional de Historia: Fuentes orales y Visuales, Iruñea-Panplona, 7-9 septiembre, 151-184.

CUESTA, R (2011). Historia con memoria y didáctica crítica. Con-ciencia Social, no 15, 15-30.

DELGADO-ALGARRA, E. J. (2013). Citizenship Education and Historical Memory: Review of Patriotism in Japanese Schools. European Scientific Journal, special number, 309-317. Recuperado de http:// hdl.handle.net/10272/7995 [17 de septiembre de 2014]

DELGADO-ALGARRA, E. J. (2014). Educación para la ciudadanía en la enseñanza de las Ciencias Sociales y su vinculación con las dimensiones de la memoria: estudio de caso en ESO. Huelva: Universidad de Huelva. (Tesis doctoral). Recuperado de http://hdl.handle.net/10272/8841 [8 de septiembre de 2014]

DÍEZ GUTIÉRREZ, E. J. (2013). La memoria histórica en los libros de texto escolares.Didáctica de las Ciencias Experimentales y Sociales, $\mathrm{n}^{\circ} 26$, 23-41. DOI: 10.7203/dces.27.2373.
ESTEPA-GIMÉNEZ, J. y DELGADOALGARRA, E. J. (2015). Educación para la Ciudadanía y memoria histórica. En B. Borghi, F. F. García Pérez y O. Moreno (Eds.), Novi Cives Cittadini dall infanzia in poi (126136). Bolonia: Pàtron Edittore.

FUJIOKA, NOBUKATSU (2000): Patología de la "visión masoquista de la historia”. Tôkyô: Bungeishunjū [藤 岡信勝 $\langle 2000\rangle$ 。自虐歴史観の病理。東 京: 文藝春秋。(En japonés)].

HABERMAS, J. (1994). Historia y crítica de la opinión pública: La transformación estructural de la vida pública. Barcelona: Gustavo Gili

HESS, D. (2002). Teaching controversial public issues discussions: Learning from skilled teachers. Theory and Research in Social Education, v. 30, $\mathrm{n}^{\circ}$ 1, 10-41. DOI: 10.1080/00933104.2014.965860.

HESS, D. (2008). Controversial issues and democratic discourse. In L. S. LEVSTIK y C. A. TYSON (eds.), Handbook of Research in Social Studies Education (59-77). New York: Taylor and Francis.

HIGURASHI, Y. (2003). Los Juicios de Tokio. Tokio: Kôdansha. [日暮吉延 $\langle 2008\rangle$ 。東京裁判。東京:講談社。(En japonés)]

IENAGA, S. (1993): The glorification of the war in the Japanese education. International Security, v. $3, \mathrm{n}^{\circ} 18$, 113-133.

KAYAMA, R. (2002). Síndrome del pequeño nacionalismo: doctrina de 
los jóvenes de Japón. Tokio: Chûô Kôron Shinsho. [香山リカ〈2002〉。ぷ ちナショナリズム症候群：若者たちのニ ツポン主義。東京：中央公論新社。(En japonés)].

KELLY, T. (1986). Discussing controversial issues: Four perspectives on the teacher's role. Theory and Research in SocialEducation, $\mathrm{n}^{\mathrm{0}} 14,113-138$.DOI: 10.1080/00933104.2014.965860.

KINGMAN, E. (2012). Usos ambiguos del archivo, la historia y la memoria. Íconos. Revista de Ciencias Sociales, $\mathrm{n}^{\circ}$ 42,123-133.http://dialnet.unirioja. es/servlet/articulo? codigo $=3817661$ [13 de octubre de 2014].

Ley Fundamental de Educación de 31 de marzo de 1947 [1947年3月31日教 育基本法。(En japonés)].

LÓPEZ, C. (2008). Crítica del entramado ético-político en la ciudadanía democrática. Con-Ciencia Social, $\mathrm{n}^{\circ}$ 12, 41-62.

MARTÍNEZ BONAFÉ, J. (2004). Esfera pública, conocimiento escolar y didáctica crítica.Con-Ciencia Social, $\mathrm{n}^{\circ} 8,51-62$.

MATTOZZI, I. (2008). Memoria y formación histórica. La memoria en la clase de Historia. Íber, $\mathrm{n}^{\circ} 55,30-42$.

MOCHIZUKI, M. M. (2010). The Yasukuni Conundrum: Japan's Contested Identity and Memory. B. SCHWARTZ and K. MIKYOUNG (eds.), Northeast Asia's Difficult Past: Essays in Collective Memory (3151). New York: Palgrave Macmillan Memory Studies.
NOZAKI, Y. (2001): Feminism, Nationalism, and the Japanese Textbook Controversy over 'Comfort Women. In W. F. TWINE and K. M. BLEE (eds.), Feminism and Antiracism: International Struggles for Justice (170-192). New York: New York University Press.

OLIVA, G. (2003). L' alibi della resisten$z a$. Ovvero come abbiamo vinto la secondaguerramondiale. Mondadori.

OTEÍZA, T. (2011). Patrones valorativos en el discurso oficial de Derechos Humanos en Chile: Dando valor al pasado y construyendo memorias históricas en la sociedad. Discurso y Sociedad, v. 4, $\mathrm{n}^{\circ}$ 1, 151-183.

PAGÉS, J. (2008) El lugar de la memoria en la enseñanza de la historia. Ïber, Didáctica de las Ciencias Sociales, Geografía e Historia, $\mathrm{n}^{\circ}$ 55, 43-53.

PAGÉS, J. y OLLER, M. (2005). Las representaciones sociales del derecho, la justicia y la ley de un grupo de adolescentes catalanes de $4^{\circ}$ de ESO. Enseñanza de las Ciencias Sociales, $\mathrm{n}^{\circ}$ 6, 3-19.

RINALDI, G. (2008). Storia e memoria. En ZIRUOLO, L (ed.), I luoghi, la storia, la memoria. Genova: Le Mani.

SHIN, G.W. (2011). History Textbooks, Divided Memories, and Reconciliation. In G.W. SHIN y D.C. SNEIDER (coords.), History Textbooks and the Wars in Asia: Divided Memories (3-20). New York: Routledge.

S/A (2013). El Primer Ministro Abe rinde culto por primera vez desde que 
asumió el cargo hace un año. Informó sobre un "juramento de paz duradera". Sankei Shimbun, Periódico Especial, December 26 [匿名の (20 01 $3)$ 。安倍首相が靖国神社に参拝 政 権 1 年 就任後初「恒久平和の誓い、 お伝えした」。産経新聞、号外新聞、12 月26日。(En japonés)]. Recuperado de http://sankei.jp.msn.com/politics/ news/131226/plc13122611340014n1.htm [16 de septiembre de 2014]. TUTIAUX-GUILLON, N. (2003). L'Histoire enseignée entre coutume disciplinaire et la formation de la conscience historique. En TUTIAUXGUILLÉN, N. (ed.), Identités, mémoires, consciente historique (2742). Saint Etienne: Université de Saint Etienne.
TODOROV, T. (2012). Mémoire du mal Tentation du bien: enquête sur le siècle. París: Robert Laffont.

TOHMATSU, H. (2011). Japanese History Texbooks in a Comparative Perspective. In G.W. SHIN and D.C. SNEIDER (coords.), History Textbooks and the Wars in Asia: Divided Memories (115-139). New York: Routledge.

VANZETTO, L. s/f. Per una nuova didatticadellastoria:L'insegnamento della storia e la costruzione sociale della memoria. Materiali di storia, $\mathrm{n}^{\mathrm{o}}$ 22, 25-32. Recuperado de http://www. centrostudiluccini.it/pubblicazioni/ materiali/22/insegnamento-storia. pdf [29 de octubre de 2014]. 
